\title{
Combined Use of Several Mitogens for Mitotic Stimulation to Human Lymphocytes
}

\author{
TOSHIO SOFUNI ${ }^{1}$, AND MICHIHIRO C. YOSHIDA ${ }^{2}$ \\ ${ }^{1}$ Division of Genetics and Mutagenesis, National Institute of Hygienic Sciences, \\ 1-18-1 Kamiyoga, Setagaya-ku, Tokyo 158, and \\ ${ }^{2}$ Chromosome Research Unit, Faculty of Science, Hokkaido University, \\ Kita-10, Nishi-8, Kita-ku, Sapporo 060
}

\section{Human lymphocyte culture/Phytohemagglutinin/Lectin/Mitogens/Mitotic index}

Several different type of mitogens, such as phytohemagglutinin (PHA) including PHA-P, - M, -W, -C, $-\mathrm{L}$ and $-\mathrm{E}+\mathrm{L}$, pokeweed mitogen (PWM), Concanavalin A (Con-A), lipopolysaccharide (LPS), wheat germ agglutinin (WGA) and soybean agglutinin (SBA) were compared their mitotic stimulating effects to human lymphocytes in single use and also combined use of these mitogens.

In the tests with single use of each mitogen, PHA was confirmed to be the most superior mitogen. Of several different types of PHA, PHA-C, $-\mathrm{L}$ and $-\mathrm{E}+\mathrm{L}$ were not appropriate to stimulate human lymphocytes, though PHA-P, $-M$ and $-W$ induced sufficiently mitoses. The most appropriate concentration of PWM was $50 \mu \mathrm{g} / \mathrm{ml}$, but the highest mitotic index reached at 5 days after the initiation of culture. Con-A did not induce sufficient mitoses, and LPS, WGA and SBA were inadequate to stimulate human lymphocytes in the single use.

In the combined use of two mitogens, there was no reliable combination to enhance mitotic stimulation. When three different mitogens were used simultaneously, only one combination with PHA-M, PWM and SBA effectively enhanced the mitotic index when compared to the single use. In the confirmatory experiment for this combination carried out on 5 different donors, the enhanced effect to mitotic index was recognized in two out of three donors with low response to PHA, but the low level of mitotic indices was seen in remaining 2 donors with high response to PHA. This finding suggests that this combination may overcome a disadvantage in some of the low responders to PHA.

\section{INTRODUCTION}

For the automation of human chromosome analyses, especially for analyses of chromosome aberrations induced by low-dose radiation exposure, the accumulation of enormous number of mitoses is thought to be a great advantage. However, reports in which the culture condition of human lymphocytes including the stimulating condition by mitogens was examined in detail, were quite limited, and the purpose of the present study is to establish the most appropriate condition in mitotic stimulation to human lymphocytes.

One of mitogens, phytohemagglutinin (PHA), has been widely used for mitotic stimulation to human lymphocytes ${ }^{1)}$, and several different types of PHA, such as PHA-P, -M, -W and others were compared its ability to induce mitoses in the present study.

It was known to exist some individual differences in response to $\mathrm{PHA}^{2)}$ and the limitation of 
only T-lymphocyte stimulation by $\mathrm{PHA}^{3)}$. Therefore, pokeweed mitogen (PWM) which was known to stimulate $\mathrm{T}$ - and B-lymphocytes ${ }^{3}$, and some other mitogens such as Concanavalin $\mathrm{A}$ (Con-A), lipopolysaccharide (LPS), wheat germ agglutinin (WGA) and soybean agglutinin (SBA) were used and compared their mitotic stimulating effects in single use and also combined use of these mitogens.

\section{MATERIALS AND METHODS}

Experiment 1: Three different types of PHA, such as PHA-P (Difco), PHA-W (Wellcome) and PHA-E + L (Vector Lab.) were used. In addition, PWM (Polysciences), Con-A (Boehringer Mannheim GmbH), WGA (Boehringer Mannheim GmbH) and LPS (Difco) were used.

A whole blood culture technique ${ }^{4)}$ was adopted in this study. $20 \mathrm{ml}$ of peripheral blood obtained from each four healthy young adults (A: 22-year-old male, B: 23-year-old male, C: 28-year-old female, D: 22-year-old female) were mixed with $180 \mathrm{ml}$ of RPMI 1640 including $10 \%$ of fetal calf serum, and divided into $10 \mathrm{ml}$ in each culture flask and then kept at $4^{\circ} \mathrm{C}$ over-night. After the addition of mitogens, the cells were cultured at $37^{\circ} \mathrm{C}$ for 50 hours including last 2 hours of Colcemid treatment $(0.05 \mu \mathrm{g} / \mathrm{ml}$ in final concentration). Exceptionally, the cultures treated with PWM and LPS incubated for 3, 5 and 6 days.

The content of one vial of PHA-P and PHA-W were dissolved with $5 \mathrm{ml}$ of sterilized distilled water and $50 \mu \mathrm{l}$ of them were added into $10 \mathrm{ml}$ of the culture $(5 \mu \mathrm{l} / \mathrm{ml}$ in final volume). The final concentration of each PHA-E $+\mathrm{L}$, Con-A, WGA, PWM and LPS was 0.25, 0.2, 2.0, 10 and $10 \mu \mathrm{g} / \mathrm{ml}$, respectively. When two different mitogens were simultaneously used, a half of the concentration for the single use was used.

Additionally, peripheral blood obtained from a donor (E: 25-year-old male) was cultured according to the same method described above, and used to find appropriate concentration in each PHA-P, PWM, Con-A and WGA, and appropriate sampling time in PHA-E $+\mathrm{L}$ using one fixed concentration.

1,000 nucleated cells including mitoses were observed under a microscope and mitotic index (\%) was recorded.

Experiment 2: Four different mitogens, PHA-M (Difco), PHA-C (Boehringer Mannheim $\mathrm{GmbH}$ ), PWM (Boehringer Mannheim GmbH) and Con-A (Boehringer Mannheim GmbH) were used. The final volume of PHA-M was 10 or $20 \mu \mathrm{l} / \mathrm{ml}$, and the final concentration of other three mitogens, PHA-C, PWM and Con-A was adjusted to be same as $10 \mu \mathrm{g} / \mathrm{ml}$.

Peripheral blood obtained from a donor (F: 30-year-old male) was cultured with RPMI1640 including $15 \%$ of fetal calf serum. The standard cultures consisted with $0.3 \mathrm{ml}$ of whole blood, $4.6 \mathrm{ml}$ of culture medium and $0.1 \mathrm{ml}$ of mitogens, though the volume of culture medium was adjusted according to the volume of mitogens to make $5 \mathrm{ml}$ of the culture in total.

When two to four different mitogens were used simultaneously, the same volume for single use of each mitogen simply added and the volume of culture medium was adjusted according to 
the total volume of mitogens to make $5 \mathrm{ml}$ of the culture in total. At 28 hours after the initiation of culture, Colcemid $(0.1 \mu \mathrm{g} / \mathrm{ml}$ in final concentration) was added and the cells were harvested at 52 hours. The mitotic index (\%) was scored in 100 or 500 nucleated cells.

Experiment 3: Five different mitogens, PHA-M (Difco), PHA-L (Vector Lab.), PWM (Polysciences), Con-A (Vector Lab.) and SBA (Vector Lab.) were used. The final volume of PHA-M in the culture was $20 \mu \mathrm{l} / \mathrm{ml}$, and the final concentration of each PHA-L, PWM, Con-A and SBA was $100,20,20$ and $200 \mu \mathrm{g} / \mathrm{ml}$, respectively.

Peripheral blood obtained from the same donor $(\mathrm{F})$ in Experiment 2 was cultured with RPMI1640 including $15 \%$ of fetal calf serum. The cultures consisted with $0.3 \mathrm{ml}$ of whole blood, $4.6 \mathrm{ml}$ of culture medium and $0.1 \mathrm{ml}$ of a mitogen. When two or three different mitogens were used simultaneously, the volume of culture medium decreased according to the total volume of mitogens. At 28 hours after the initiation of culture, Colcemid $(0.1 \mu \mathrm{g} / \mathrm{ml}$ in final concentration) was added and the cells were harvested at 52 hours. The mitotic index (\%) was scored in 500 nucleated cells.

Experiment 4: Three different mitogens, PHA-W (Wellcome), PWM (GIBCO) and SBA (Vector Lab.) were used. The final volume of PHA-W was 10 or $20 \mu \mathrm{l} / \mathrm{ml}$, and the final concentration of PWM and SBA was $20 \mu \mathrm{g} / \mathrm{ml}$ and $200 \mu \mathrm{g} / \mathrm{ml}$, respectively.

Peripheral blood obtained from five donors (G: 26-year-old male, H: 22-year-old male, I: 21-year-old female, J: 22-year-old female and $\mathrm{K}$ : 22-year-old male) was used. $0.3 \mathrm{ml}$ of whole blood was added to $4.7 \mathrm{ml}$ of culture medium and kept at $4^{\circ} \mathrm{C}$ over-night. After the addition of mitogens $(20 \mu \mathrm{l} / \mathrm{ml}$ of PHA, $20 \mu \mathrm{l} / \mathrm{ml}$ of PWM, 100 or $200 \mu \mathrm{g} / \mathrm{ml}$ of SBA), the cultures were incubated at $37^{\circ} \mathrm{C}$ for 28 hours and treated with Colcemid $(0.1 \mu \mathrm{g} / \mathrm{ml}$ in final concentration), and then the cells were harvested at 52 hours. The mitotic index $(\%)$ was scored in 1,000 nucleated cells.

\section{RESULTS}

Experiment 1: The results obtained from four different donors are present in Table 1. PHA-P $(5 \mu \mathrm{l} / \mathrm{ml})$ induced mitoses with almost same extent among four donors. PHA-W $(5 \mu \mathrm{l} /$ $\mathrm{ml}$ ) induced mitoses with same degree to that of PHA-P in one donor (D), but in other three donors the mitotic indices were lower than that of PHA-P. When $10 \mu \mathrm{l} / \mathrm{ml}$ of PHA-W was used in the donor $\mathrm{D}$, a higher mitotic index was obtained, suggesting that the appropriate concentration of PHA-W was $10 \mu \mathrm{l} / \mathrm{ml}$.

Con-A $(0.2 \mu \mathrm{g} / \mathrm{ml})$ induced mitoses with a low rate when compared to that of PHA-P or PHA-W, and almost no mitosis was observed in PHA-E $+\mathrm{L}$ and WGA.

For PWM and LPS, the mitotic indices were scored in 3-, 5- and 6-day cultures using one fixed concentration. PWM induced mitoses with relatively high degree in all four donors, and the maximum value of average mitotic indices was obtained in the 5-day culture. On the other hand, LPS induced almost no mitosis in all cultures. 
Table 1. Mitotic stimulation of seven different mitogens in whole blood cultures from four different donors

\begin{tabular}{|c|c|c|c|c|c|c|c|}
\hline \multirow{2}{*}{ Mitogen } & \multirow{2}{*}{$\begin{array}{c}\text { Dose } \\
(\mu \mathrm{g} / \mathrm{ml})^{*}\end{array}$} & \multirow{2}{*}{$\begin{array}{c}\text { Culture } \\
\text { time (h) }\end{array}$} & \multicolumn{5}{|c|}{ Mitotic indices $(\%)$ in different donors } \\
\hline & & & A & B & C & $\mathrm{D}$ & Average \\
\hline PHA-P & 5.0 & 50 & 3.2 & 2.2 & 2.4 & 3.5 & 2.83 \\
\hline \multirow[t]{2}{*}{ PHA-W } & 5.0 & 50 & 1.1 & 1.1 & 0.6 & 3.3 & 1.53 \\
\hline & 10.0 & 50 & $\mathrm{NT}^{* *}$ & NT & NT & 5.2 & $(5.2)$ \\
\hline Con-A & 0.2 & 50 & 0.2 & 0.2 & 0.0 & 1.1 & 0.38 \\
\hline PHA-E + L & 0.25 & 50 & 0.0 & 0.1 & 0.0 & 0.0 & 0.03 \\
\hline \multirow[t]{3}{*}{ WGA } & 0.2 & 50 & $N^{\prime} \mathrm{T}$ & 0.0 & NT & NT & $(0.0)$ \\
\hline & 2.0 & 50 & 0.0 & 0.0 & 0.0 & 0.1 & 0.03 \\
\hline & 10.0 & 50 & NT & 0.0 & NT & NT & $(0.0)$ \\
\hline \multirow[t]{3}{*}{ PWM } & 10.0 & 72 & 2.0 & 1.3 & 6.2 & 5.4 & 3.73 \\
\hline & 10.0 & 120 & 3.9 & 2.4 & 6.2 & 5.5 & 4.50 \\
\hline & 10.0 & 144 & 2.2 & 3.7 & 4.2 & 3.5 & 3.40 \\
\hline \multirow[t]{3}{*}{ LPS } & 10.0 & 72 & 0.0 & 0.0 & 0.1 & 0.1 & 0.05 \\
\hline & 10.0 & 120 & 0.0 & 0.0 & 0.0 & 0.0 & 0.0 \\
\hline & 10.0 & 144 & 0.0 & NT & 0.0 & $\mathrm{NT}$ & 0.0 \\
\hline PHA-P + PHA-W & $2.5+2.5$ & 50 & 2.1 & 2.6 & 1.8 & 5.1 & 2.90 \\
\hline PHA-P + PHA-E $+\mathbf{L}$ & $2.5+0.125$ & 50 & 2.8 & 1.5 & 2.2 & 4.7 & 2.80 \\
\hline PHA-P + Con-A & $2.5+0.1$ & 50 & 2.7 & 1.1 & 2.5 & 6.5 & 3.20 \\
\hline PHA-P + WGA & $2.5+1.0$ & 50 & 1.8 & 1.3 & 0.0 & 6.4 & 2.38 \\
\hline $\mathrm{PHA}-\mathrm{W}+\mathrm{PHA}-\mathrm{E}+\mathrm{L}$ & $2.5+0.125$ & 50 & 0.3 & 0.7 & 0.2 & 2.2 & 0.85 \\
\hline PHA-W + Con-A & $2.5+0.1$ & 50 & 0.0 & 0.2 & 0.0 & 1.3 & 0.38 \\
\hline PHA-W + WGA & $2.5+1.0$ & 50 & 0.1 & 0.2 & 0.0 & 2.3 & 0.65 \\
\hline Con-A+PHA-E +L & $0.1+0.125$ & 50 & 0.0 & 0.2 & 0.0 & 0.7 & 0.23 \\
\hline PHA-E + L + WGA & $0.125+1.0$ & 50 & 0.0 & 0.0 & 0.0 & 0.0 & 0.0 \\
\hline
\end{tabular}

* In PHA-P and PHA-W, the content of one vial was dissolved with $5 \mathrm{ml}$ of sterilized water and the final volume $(\mu \mathrm{l} / \mathrm{ml})$ is shown.

** NT: Not tested.

When two different mitogens were used simultaneously, no enhancement was observed in any combination of two mitogens used: only same extent of mitotic induction was observed in the combination using PHA-P, and all other combinations showed low mitotic indices or almost no mitotic induction.

The results on the test for concentration-dependency of several mitogens using peripheral blood of a donor (E) are shown in Table 2. The mitotic index by PHA-P reached to the maximum at the lowest concentration of $5 \mu \mathrm{l} / \mathrm{ml}$, and then mitotic index decreased according to increasing concentration. A similar tendency was obtained in Con-A: the maximum mitotic index was seen at the lowest concentration, but its value was half of that of PHA-P.

The mitotic index in the 4-day culture with PWM, increased according to increasing concentration and reached to the maximum at $50 \mu \mathrm{g} / \mathrm{ml}$ and then decreased at the highest 
Table 2. Mitotic stimulation of five different mitogens in whole blood cultures from a donor

\begin{tabular}{lccc}
\hline Mitogen & $\begin{array}{c}\text { Dose } \\
(\mu \mathrm{g} / \mathrm{ml})^{*}\end{array}$ & $\begin{array}{c}\text { Culture } \\
\text { time }(\mathrm{h})\end{array}$ & $\begin{array}{c}\text { Mitotic indices }(\%) \\
\text { in the donor } \mathrm{E}\end{array}$ \\
\hline PHA-P & 5.0 & 50 & 1.5 \\
& 10.0 & 50 & 1.1 \\
& 20.0 & 50 & 0.5 \\
Con-A & 100.0 & 50 & 0.1 \\
& 1.0 & 50 & 0.8 \\
& 10.0 & 50 & 0.5 \\
PWM & 20.0 & 50 & 0.4 \\
& 50.0 & 50 & 0.1 \\
& 10.0 & 96 & 0.5 \\
& 25.0 & 96 & 2.5 \\
WGA & 50.0 & 96 & 4.6 \\
& 100.0 & 96 & 3.3 \\
PHA-E $+\mathrm{L}$ & 50.0 & 50 & 0.0 \\
& 100.0 & 50 & 0.0 \\
& 0.25 & 50 & 0.0 \\
& 0.25 & 52 & 0.0 \\
& 0.25 & 54 & 0.1 \\
& 0.25 & 56 & 0.0 \\
\hline
\end{tabular}

* In PHA-P, the final volume $(\mu \mathrm{l} / \mathrm{ml})$ in the culture is shown.

concentration.

Two different concentrations were used for WGA, but there was no mitotic induction. For PHA-E $+\mathrm{L}$, several different sampling times were used at $0.25 \mu \mathrm{g} / \mathrm{ml}$ (the appropriate concentration indicated by a instruction was $0.2 \mu \mathrm{g} / \mathrm{ml}$ ), but almost no mitotic induction was seen in any sampling time.

Experiment 2: Peripheral blood obtained from a donor $(F)$ was used for four different mitogens in the single use and combined use. PHA-M induced sufficient mitoses with almost same degree in two concentrations ( 10 and $20 \mu \mathrm{l} / \mathrm{ml}$ ), but PHA-C showed a quite low value of the mitotic index and no mitotic induction was seen in PWM and Con-A (Table 3).

When two mitogens were used simultaneously, no enhancement of mitotic induction was seen in any combination. Two combinations, PHA-M+PHA-C and PHA-M+Con-A, showed almost same degree of the mitotic index as that of single use of PHA-M, and in other combination low mitotic indices were obtained.

The combination of three or four mitogens also showed no remarkable enhancement of mitotic induction. An almost same degree of mitotic induction as seen in the single use of PHA-M was obtained in two different combinations of three mitogens (PHA-M+PHA-C+ 
Table 3. Mitotic stimulation of four different mitogens in whole blood cultures from a donor

\begin{tabular}{lccc}
\hline Mitogen & $\begin{array}{c}\text { Dose } \\
(\mu \mathrm{g} / \mathrm{ml})^{*}\end{array}$ & $\begin{array}{c}\text { Culture } \\
\text { time }(\mathrm{h})\end{array}$ & $\begin{array}{c}\text { Mitotic indices }(\%) \\
\text { in }\end{array}$ \\
\hline the donor $\mathrm{F}^{* *}$
\end{tabular}

* In PHA-M, the final volume $(\mu 1 / \mathrm{ml})$ in the culture is shown.

** Mitotic indices in 100 cells are shown. Parentheses indicate mitotic indices in 500 cells.

PWM and PHA-M+PHA-C + Con-A) and in the combination of four mitogens, but mitotic indices in other combinations were lower than that of the single use of PHA-M.

Experiment 3: Five different mitogens were tested using peripheral blood of the same donor $(\mathrm{F})$ in the Experiment 2. In the single use, PHA-M was superior to any other mitogens. Three mitogens, PHA-L, PWM and Con-A showed low mitotic indices and SBA had no mitosis (Table 4).

In the use of two mitogens, the combination of PHA-M +other mitogens and PHA-L + other mitogens were mainly examined. In the former combination, PHA-M+PWM showed a same activity to that of the single use of PHA-M, but other combinations showed an inhibitory effect to PHA-M. In the latter, all combinations showed almost same degree as that of the single use.

When three mitogens were used simultaneously, the combination of PHA-M + PWM + SBA showed a remarkable enhancement to the mitotic induction of the single use of PHA-M, and its mitotic index was almost twice that of the single use. Two combinations, PHA-M+PHA-L + Con-A and PHA-M+PWM + Con-A showed an almost same activity to that of the single use, but other three combinations showed low mitotic indices.

Experiment 4: Five donors $(\mathrm{G}-\mathrm{K})$ were subjected to the experiment using three different 
Table 4. Mitotic stimulation of five different mitogens in whole blood cultures from a donor

\begin{tabular}{lccc}
\hline Mitogen & $\begin{array}{c}\text { Dose } \\
(\mu \mathrm{g} / \mathrm{ml})^{*}\end{array}$ & $\begin{array}{c}\text { Culture } \\
\text { time }(\mathrm{h})\end{array}$ & $\begin{array}{c}\text { Mitotic indices (\%) } \\
\text { in the donor } \mathrm{F}\end{array}$ \\
\hline PHA-M & 20 & 52 & 6.5 \\
PHA-L & 100 & 52 & 1.8 \\
PWM & 20 & 52 & 1.1 \\
Con-A & 20 & 52 & 0.7 \\
SBA & 200 & 52 & 0.0 \\
PHA-M+PHA-L & $20+100$ & 52 & 1.3 \\
PHA-M+PWM & $20+20$ & 52 & 6.2 \\
PHA-M+Con-A & $20+20$ & 52 & 2.8 \\
PHA-M+SBA & $20+200$ & 52 & 2.6 \\
PHA-L+PWM & $100+20$ & 52 & 1.1 \\
PHA-L+Con-A & $100+20$ & 52 & 2.3 \\
PHA-L+SBA & $100+200$ & 52 & 2.3 \\
PHA-M + PHA-L+PWM & $20+100+20$ & 52 & 2.0 \\
PHA-M +PHA-L+Con-A & $20+100+20$ & 52 & 7.1 \\
PHA-M + PHA-L+SBA & $20+100+200$ & 52 & 1.7 \\
PHA-M + PWM+Con-A & $20+20+20$ & 52 & 6.2 \\
PHA-M + PWM+SBA & $20+20+200$ & 52 & 14.3 \\
PHA-M +Con-A+SBA & $20+20+200$ & 52 & 3.9 \\
\hline
\end{tabular}

* In PHA-M, the final volume $(\mu \mathrm{l} / \mathrm{ml})$ in the culture is shown.

Table 5. Mitotic stimulation of three different mitogens in whole blood cultures from five different donors

\begin{tabular}{|c|c|c|c|c|c|c|c|c|}
\hline \multirow{2}{*}{ Mitogen } & \multirow{2}{*}{$\begin{array}{c}\text { Dose } \\
(\mu \mathrm{g} / \mathrm{ml})^{*}\end{array}$} & \multirow{2}{*}{$\begin{array}{l}\text { Culture } \\
\text { time }(\mathrm{h})\end{array}$} & \multicolumn{6}{|c|}{ Mitotic indices $(\%)$ in different donors } \\
\hline & & & $\mathrm{G}$ & $\mathbf{H}$ & I & $\mathrm{J}$ & $\mathbf{K}$ & Average \\
\hline \multirow[t]{2}{*}{ PHA-W } & 20 & 52 & 1.6 & 1.2 & 3.4 & 4.3 & 1.8 & 2.5 \\
\hline & 10 & 52 & 1.2 & $\mathrm{NT}^{* *}$ & NT & NT & NT & $(1.2)$ \\
\hline PHA-W + PWM & $20+20$ & 52 & 1.4 & 0.9 & 2.1 & 2.1 & 1.2 & 1.5 \\
\hline \multirow[t]{2}{*}{$\mathrm{PHA}-\mathrm{W}+\mathrm{PWM}+\mathrm{SBA}$} & $20+20+200$ & 52 & 3.5 & 2.7 & 2.6 & 3.5 & 1.9 & 2.8 \\
\hline & $20+20+100$ & 52 & 2.5 & NT & NT & NT & NT & $(2.5)$ \\
\hline
\end{tabular}

* In PHA-W, the final volume $(\mu \mathrm{l} / \mathrm{ml})$ is shown.

** NT: Not tested.

mitogens (PHA-W, PWM and SBA) for the confirmation of the enhanced effect observed in Experiment 3. In the single use of $20 \mu \mathrm{l} / \mathrm{ml}$ of PHA-W, the highest mitotic index was seen in the donor $\mathrm{J}$ and the lowest was seen in the donor $\mathbf{H}$, and its difference was 3.8 fold. In $10 \mu \mathbf{l} /$ $\mathrm{ml}$ of PHA-W the mitotic index was slightly lower than that of $20 \mu \mathrm{l} / \mathrm{ml}$ of PHA-W (Table 5).

The combination of PHA-W and PWM showed always lower mitotic indices than that single use of PHA-W in five donors. On the other hand, the combination of three mitogens 
$(\mathrm{PHA}-\mathrm{W}+\mathrm{PWM}+\mathrm{SBA})$ showed an enhancement of the mitotic induction in two donors ( $\mathrm{G}$ and $\mathrm{H}$ ), and its mitotic indices were more than twice that of single use in both donors. However, in other three donors, no remarkable enhancement to the mitotic induction was seen: an almost same mitotic index was seen in the donor $\mathrm{K}$, and lower mitotic indices were seen in the donors $\mathrm{I}$ and $J$. When the low concentration of SBA was used in this combination on the donor $G$, the mitotic index slightly decreased.

\section{DISCUSSION}

The results obtained in the present study indicate that PHA was superior than any other mitogens to stimulate mitoses in human peripheral blood cultures. Among several different types of PHA, PHA-P, PHA-M and PHA-W showed a high quality for mitotic induction, but to find the most appropriate condition on these three and the most suitable one among them, further experiments are needed. On the other hand, PHA-L, PHA-E + L and PHA-C showed remarkably low mitotic indices or almost no mitotic induction, and these were thought to be useless for the mitotic stimulation in human peripheral blood culture.

The present study demonstrated that PWM could sufficiently stimulate mitoses only in late culture time such as 4 to 6 days, and this finding suggest the limited use of PWM especially in simultaneous stimulation with PHA, because PHA induced mitoses in early culture time. For the analysis of chromosomal aberrations induced by ionizing irradiation, only first mitoses should be examined in early culture time (about 2 days) to avoid mitotic selection of cells having aberrations $s^{5)}$, and the elongation of culture time might produce contamination of second or third mitoses.

Con-A could induce mitoses, but its extent was insufficient, and other mitogens, such as LPS, WGA and SBA, could not induce mitoses, indicating these mitogens were inadequate to stimulate human lymphocytes in the single use.

In the tests with combined use of two mitogens, any combination studied here could not enhance mitotic stimulation and almost same level or low level of mitotic indices were observed when compared to the single use.

When three different mitogens were used simultaneously, only one combination with PHA-M, PHA and SBA effectively enhanced the mitotic stimulation when compared to that of the single use of PHA-M. The confirmatory experiment for this combination with PHA-W was carried out using peripheral blood obtained from 5 different donors (G-K). The enhanced effect of mitotic stimulation was recognized in two donors ( $\mathrm{G}$ and $\mathrm{H}$ ) and the mitotic indices in the three combination were more than twice that of the single use of PHA-W. Another donor (K) showed an almost same level of mitotic indices in the single and three-combined use. In remaining two donors ( $\mathrm{H}$ and $\mathrm{I}$ ), the mitotic indices in the three combination were relatively lower than that of the single use.

The mitotic induction by PHA-W in three donors ( $\mathrm{G}, \mathrm{H}$ and $\mathrm{K}$ ) were remarkably lower than that of two donors ( $\mathrm{H}$ and $\mathrm{I}$ ), indicating these three donors being low responders to PHA-W. The enhanced effect of mitotic stimulation by the three combination was observed in 
two of three low responders to PHA-W. This finding suggests that three-combined use with PHA-W (or PHA-M as seen in Experiment 3), PWM and SBA might be effective to enhance mitotic stimulation in the individuals being less sensitive to PHA-W or PHA-M. However, the donor $\mathrm{K}$ who was also less sensitive to PHA-W showed no enhancement. Therefore, additional experiments are necessary to establish the most appropriate condition in combined use of different mitogens.

\section{REFERENCES}

1. Nowell, P. C. (1960) Phytohemagglutinin: A initiator of mitosis in cultures of normal human leukocytes, Cancer Res. 20: 462-466.

2. Pisciotta, A. V., Westring, D. W., Deprey, C. and Walsh, B. (1967) Mitogenic effect of phytohaemagglutinin at different ages, Nature 215: 193-194.

3. Sharon, N. and Lis, H. (1989) Lectins, Chapman and Hall, London and New York.

4. Hungerford, D. A. (1965) Leukocytes cultured from small inocula of whole blood and the preparation of metaphase chromosomes by treatment with hypotonic KCl, Stain Tech. 40: 333-338.

5. Sasaki, M. S. and Norman, A. (1966) Proliferation of human lymphocytes in culture. Nature 210: 913914. 